\title{
Development and Optimization of a Pulsed Electroacoustic System with Temperature Controlled Electrodes
}

\author{
R. Hussain ${ }^{1} \&$ V. Hinrichsen ${ }^{1}$ \\ ${ }^{I}$ Technische Universität Darmstadt, High-Voltage Laboratories, Darmstadt, Germany \\ hussain@hst.tu-darmstadt.de
}

\begin{abstract}
This work presents the important steps in developing and optimizing a Pulsed Electroacoustic (PEA) system. The aim is to investigate silicone rubbers (LSR) with different amount of nanofillers. Therefore, the influence of the nanofillers on the acoustic behavior such as the acoustic impedance and the speed of sound are investigated. A detailed description of the acoustic detection unit is provided. To allow the measurement at different absolute temperatures and also at temperature gradients the electrodes are temperature controlled. Reference measurements are performed to give a better and more accurate temperature profile estimation inside the samples. The influence of temperature on the attenuation and dispersion factors of different LSR samples is outlined. A final procedure for signal processing is presented to determine the correct space charge distribution.
\end{abstract}

\section{Introduction}

High voltage direct current (HVDC) power transmission is becoming increasingly competitive to high voltage alternating current (HVAC) power transmission, especially for bulk power transmission over long distances or large overlay systems. For crossing wide metropolitan areas or long distances in the open sea, especially HVDC cable lines are attractive. Regarding the insulation extruded (XLPE) HVDC cables offer significant advantages over cables of other insulation types, e.g. oil-paper insulated cables. In addition to higher permissible conductor temperatures, also jointing of extruded cables is easier. Nevertheless, in HVDC insulation systems the electric field distribution is much more complex than in AC systems, because it strongly depends on the (temperature dependent) conductivities of the insulation. Especially the presence of space charges can lead to an increased electric field stress up to the failure of the device $[1,2]$. Due to the mentioned challenges, it is significant to develop new insulating materials that are suitable especially for HVDC applications. One promising way is to introduce nanofillers into the polymer matrix for space charge suppression. Regarding cable insulation, numerous publications exist reporting on the space charge behavior of XLPE with and without nanofillers. Just very little has been published on the materials used for cable joints, which is preferably silicone rubber (LSR) because of its outstanding mechanical and electrical properties. Nowadays the most commonly used technique for space charge measurement is the Pulsed Electroacoustic method (PEA). Especially for silicone rubbers several requirements on the PEA setup itself and on the signal post processing must be considered. The purpose of this paper is thus to present a PEA setup suitable for the investigation of silicone rubbers and a proper signal post processing method. The challenges and requirements are outlined, and the finally applied optimized solutions are discussed. Additionally, the goal is to investigate the influence of nanoscale Carbon Black (CB) for different filler concentrations. A new electrode configuration for the PEA system will be introduced allowing to heat and to cool the electrodes. The goal is to simulate a more realistic temperature distribution within the sample and to analyze the temperature influence on the space charge behavior. The final aim is to better understand the charge transport mechanism inside composite materials with nanofillers with the help of the applied measurement methods and achieved measurement results.

\section{General PEA setup}

The realized, final PEA setup is shown in Figure 1. The top electrode unit consists of the $\mathrm{HV}$ electrode $E l_{1}$, an outer PVC insulation and a hole for the liquid cooling, which is provided by a LAUDA bath thermostat. In parallel to the sample $S$ a DC voltage is provided by a $\pm 30 \mathrm{kV}$ HEINZINGER PNChp voltage source. A protecting resistor $R_{\mathrm{DC}}$ is placed in series to the DC source to limit the current in the case of a breakdown and to protect the DC source from the pulse voltage. The pulse voltage is provided by a Line Type Pulse Generator (LTPG) which is connected to the electrode $E l_{1}$ via a coupling capacitor $C_{\mathrm{c}}$ and is described in chapter 2.1 .

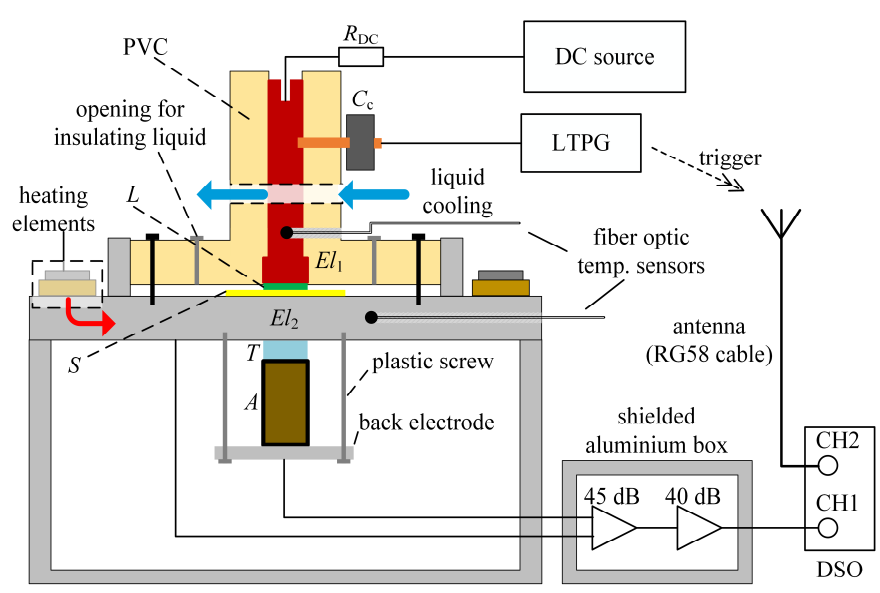

Figure 1: General PEA setup 
To ensure a good acoustic matching between the sample $S$ and the electrode $E l_{1}$ a conductive layer of fluorosilicone $L$ is placed between them. The acoustic impedance of fluorosilicone is in the range of the tested LSR samples and avoids high reflections of the acoustic waves. In addition fluorosilicone is chemical resistant against silicone oil which is used between each layer to avoid any air gaps. The ground electrode $E l_{2}$ is the cover of an aluminium box containing the acoustic detection unit. It is described in detail in chapter 2.2. Several proportionalcontrolled heating elements DN515-1228 from ThermOptics are mounted on the surface of the electrode $E l_{2}$ allowing easy heating without any liquids near the sensitive acoustic detection unit, which in general consists of the PVDF-TrFe transducer $T$, the PMMA absorber $A$ and a back electrode. The signal strength from the transducer is in the range of several $100 \mu \mathrm{V}$ and has to be amplified, so that it can be detected by the Digital Storage Oscilloscope (DSO). Two low noise amplifiers are used to achieve a total gain of $85 \mathrm{~dB}$. The first amplifier ABL0300-004030 is from WENTEQ with a gain of $45 \mathrm{~dB}$ and a bandwidth of $9 \mathrm{kHz}$ to $3 \mathrm{GHz}$. The second amplifier HD28082 from $\mathrm{HC}$ Communications has a gain of $40 \mathrm{~dB}$ and a bandwidth from $10 \mathrm{kHz}$ to $500 \mathrm{MHz}$. Both amplifiers are placed in a shielded aluminium box to avoid any influence from external interference such as generated by the pulse voltage generator. The noise generated by the LTPG is used as a trigger signal for the DSO. For this purpose a common RG58 coaxial cable is placed near the LTPG to pick up the noise (see Figure 1).

\subsection{Pulse voltage generator}

To stimulate the charge layers inside the sample a very fast pulsed electric field is needed that causes a Coulomb force. Due to the force acting on the charge, acoustic waves are generated and transferred to the piezoelectric transducer. The pressure waves $p$ are described in general by (1):

$$
p(x, t)=\rho(x) \Delta \lambda E_{\mathrm{p}}(t)
$$

where $\rho(x)$ is the charge density, $\Delta \lambda$ the width of the charge layer and $E_{\mathrm{p}}(t)$ the pulse electric field strength. From (1) it results that the amplitude of the pressure waves depends linearly on the applied pulse voltage. Due to that the shape of the pulse voltage is essential for the resolution of the PEA system. In this work the pulse voltage is realized with a Line Type Pulse Generator (LTPG). The main concept is described in detail in $[3,4]$. Basically, a coaxial cable of a specific length is charged by a DC voltage and discharged by switching a mercury reed relay. The main issue of a LTPG is that the sample impedance does not match the line impedance of $50 \Omega$. This problem can be solved by using a T-matching circuit as depicted in Figure 2 [3].

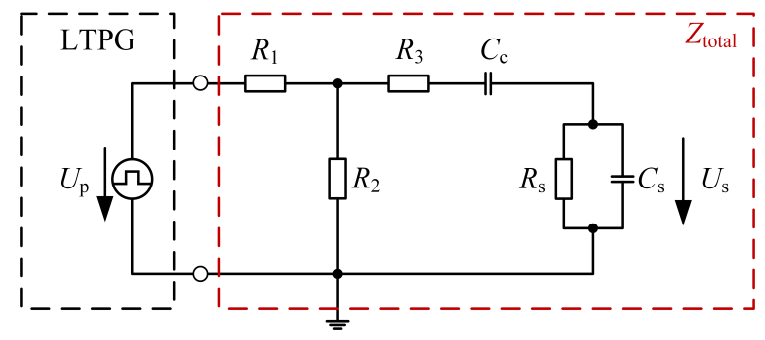

Figure 2: General setup of the T-matching circuit for the LTPG
The impedance matching circuit consists of three resistors $R_{1}, R_{2}$ and $R_{3}$. The goal is to achieve an overall impedance of $50 \Omega$ including the coupling capacitor $C_{\mathrm{c}}$ and the sample impedance represented by an equivalent $R C$-circuit in Figure 2. The sample resistance $R_{\mathrm{s}}$ is higher than $1 \mathrm{G} \Omega$ and is neglected in the further calculation. The sample capacitance $C_{\mathrm{s}}$ is around $10 \mathrm{pF}$ for all observed filler concentrations in this work and a $500 \mathrm{pF}$ coupling capacitor is considered to be sufficient. It is important that $C_{\mathrm{c}}$ is at least ten times bigger than $C_{\mathrm{s}}$ to ensure that nearly the full pulse voltage is applied to the sample [5]. For the components in the T-matching circuit in this work the following values are used: $R_{1}=10 \Omega, R_{2}=61 \Omega$ and $R_{3}=50 \Omega$. Using a $1 \mathrm{~m}$ RG58 coaxial cable for the LTPG gives a pulse width of approx. $10 \mathrm{~ns}$ [4]. The final pulse voltage is measured with a LSR sample placed between the electrodes and is shown in Figure 3. Its width is around $11 \mathrm{~ns}$ and shows only minor oscillations, indicating that the T-matching circuit fulfills its purpose.

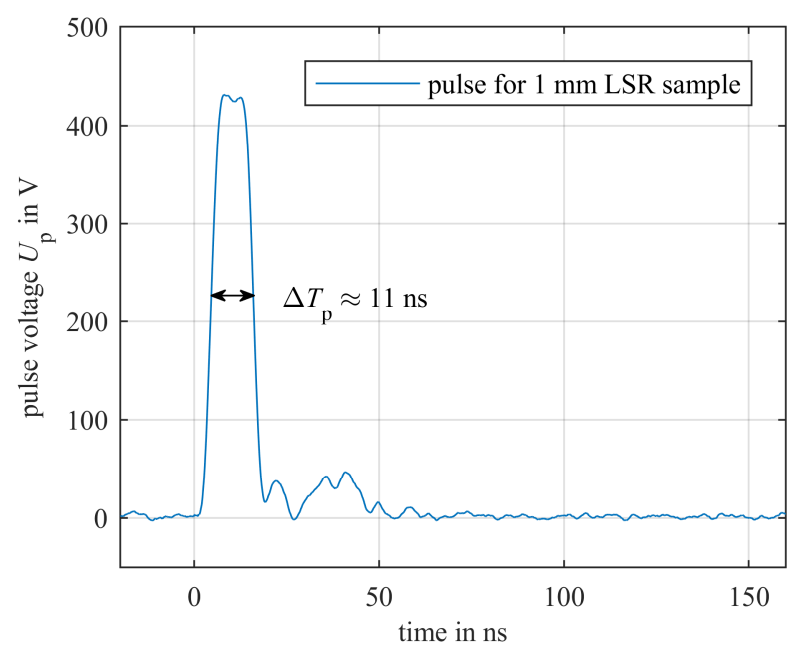

Figure 3: Finally achieved pulse voltage

\subsection{Resolution of the PEA system}

For a PEA system it is important to optimize the system for its absolute and its relative resolution. The absolute resolution $\Delta l$ is calculated according to (2) and yields the spatial resolution.

$$
\Delta l=v_{\mathrm{s}} \cdot \Delta T_{\mathrm{p}}
$$

where $v_{\mathrm{s}}$ is the speed of sound inside the sample and $\Delta T_{\mathrm{p}}$ the width of the applied pulse voltage. For the investigated LSR samples the speed of sound was determined with an ultrasonic measurement system UKS-D from GEOTRON and is for all samples around $1100 \mathrm{~m} / \mathrm{s}$. With the pulse width from Figure 3 the absolute resolution is around $12 \mu \mathrm{m}$. This value is not meaningful without knowing the actual thickness of the sample. It is better to give the relative resolution $\eta_{\mathrm{r}}$ in \% according to (3):

$$
\eta_{\mathrm{r}}=\frac{\Delta T_{\mathrm{p}}}{d_{\mathrm{s}} / v_{\mathrm{s}}} \cdot 100 \%
$$

Where $d_{\mathrm{s}}$ is the sample thickness. The relative resolution takes the thickness of the sample into account, which is more convenient. In Figure 4 the relative resolution is depicted in dependence of the sample thickness, showing that $\eta_{\mathrm{r}}$ improves for increasing $d_{\mathrm{s}}$. The minimum thickness investigated in this 
work is around $500 \mu \mathrm{m}$, which ensures a relative spatial resolution $<2.5 \%$ for all samples. This value is in good agreement with the suggestions given in [5].

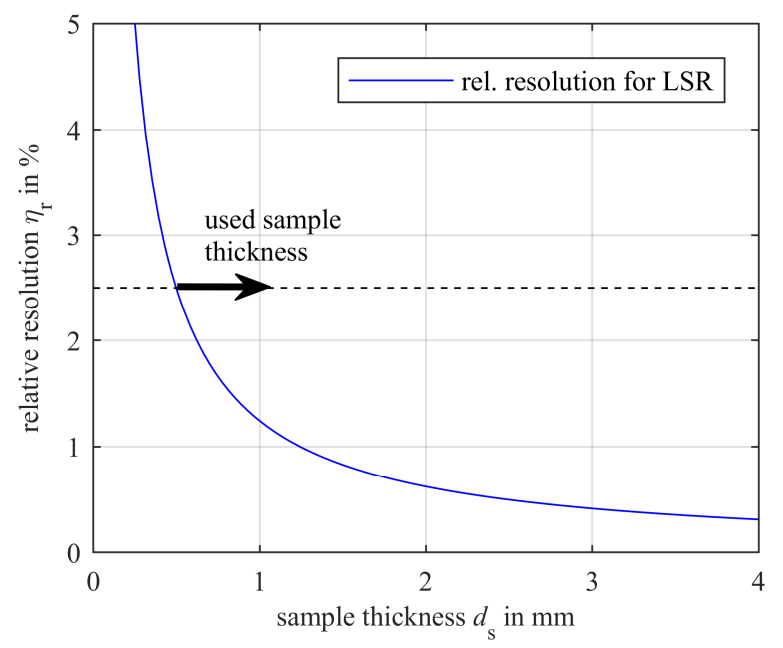

Figure 4: Relative resolution of the PEA system in dependence of the sample thickness for a pulse width of $11 \mathrm{~ns}$

\subsection{Acoustic detection unit}

The acoustic detection unit of the PEA system is shown in Figure 5 and consists of the ground electrode $E l_{2}$, the piezoelectric transduce $T$, the absorber $A$ and the back electrode. The electrode $E l_{2}$ acts as a delay line to ensure that the main signal from the sample is not influenced by the trigger signal or any reflections and has, therefore, a thickness of $30 \mathrm{~mm}$. On the backside of $E l_{2}$ a PVDF-TrFe transducer from PIEZOTECH with a thickness of $20 \mu \mathrm{m}$ is used. The transducer is metallized on both sides with a very thin layer of Cupper/Chrome and is suitable for temperatures up to at least $100{ }^{\circ} \mathrm{C}$. For the acoustic matching a PMMA absorber is used that has nearly the same acoustic impedance as the transducer.

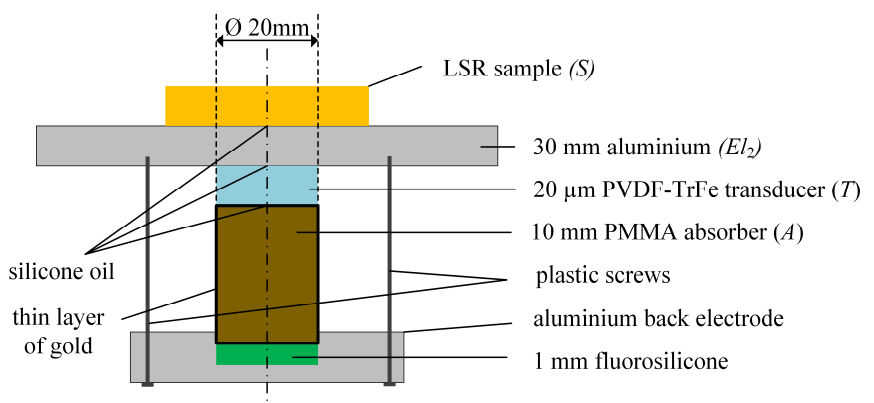

Figure 5: Acoustic detection unit of the PEA system

To avoid reflections within the absorber a thickness of $10 \mathrm{~mm}$ is sufficient. More detailed explanations in selecting a proper thickness of $E l_{2}$ and $A$ is given in [4]. To connect the transducer to the amplifier the PMMA absorber is metallized with a very thin layer of gold and is centred by an aluminium back electrode. The back electrode has a central circular clearance to align the absorber and the transducer to the HV electrode $E l_{1}$. In addition a layer of conductive fluorosilicone is placed inside the clearance to give a good and constant contact pressure. The back electrode is fixed to the electrode $E l_{2}$ by plastic screws. To avoid any air gaps between the layers a small amount of silicone oil with a kinematic viscosity around $300 \mathrm{~mm}^{2} / \mathrm{s}$ is used (see Figure 5).

\subsection{Final parameter of the PEA setup}

In this chapter the final implemented components and realized parameters of the PEA system are summarized. A very important property is the acoustic impedance of each component to avoid any reflections that would result in a distortion of the signal. For this purpose all relevant materials were investigated with an ultrasonic measurement system giving the results in Table 1.

\begin{tabular}{|c|c|c|c|}
\hline Material & $\begin{array}{c}v_{i} \text { in } \\
\mathrm{m} / \mathrm{s}\end{array}$ & $\begin{array}{c}\delta_{i} \text { in } \\
\mathrm{kg} / \mathrm{m}^{3}\end{array}$ & $\begin{array}{c}Z_{i} \text { in } \\
10^{6} \cdot \mathrm{kg} / \mathrm{m}^{2} \mathrm{~s} \\
\end{array}$ \\
\hline Aluminium $(A l)$ & 6220 & 2700 & 16.8 \\
\hline Brass $(B)$ & 3930 & 8100 & 31.8 \\
\hline $\operatorname{PVDF}(T)$ & 2222 & 1780 & 3.96 \\
\hline $\operatorname{PMMA}(A)$ & 2778 & 1180 & 3.28 \\
\hline LSR $1(S)$ & 1130 & 1068 & 1.21 \\
\hline LSR $2(S)$ & 1090 & 1063 & 1.16 \\
\hline
\end{tabular}

The results in Table 1 show that the acoustic impedance of metals is very high compared to the other materials and that PMMA has nearly the same impedance as PVDF, making it a good choice for the absorber. Furthermore, neat LSR samples (LSR 1) and LSR filled with nanoscaled Carbon Black (LSR 2) were tested. The amount of nanofillers for all tested LSR samples are below the percolation threshold, and the influence on the acoustic impedance is negligible. The final pulse voltage from the LTPG has a pulse width of around $10 \mathrm{~ns}$ with a maximum peak voltage of $500 \mathrm{~V}$ that is limited by the used mercury reed relay with a maximum switching voltage of $1 \mathrm{kV}$. With the realised pulse voltage samples between 0.5 to $2 \mathrm{~mm}$ can be analysed without any restrictions. To avoid any electromagnetic interference caused by the LTPG the piezoelectric transducer together with the acoustic detection unit are placed inside an aluminium box. It has become apparent to use special EMC shielding tape has to be used to achieve proper sealing between the electrode $E l_{2}$ and the aluminium box. The same countermeasure is applied to the external aluminium box containing the amplifiers. Placing the amplifiers in special boxes outside the main box eliminates any influence of the temperature rise in the electrode $E l_{2}$ during the measurements. To allow space charge measurements under different temperatures or temperature gradients the electrodes $E l_{1}$ and $E l_{2}$ are temperature controlled. The electrodes are described in chapter 3.

\section{Temperature controlled electrodes}

In order to investigate the influence of temperature on the space charge behavior in LSR samples the main electrodes $E l_{1}$ and $E l_{2}$ are modified as shown in Figure 1. The temperatures of both electrodes are continuously measured during the measurement. In order to have a more accurate estimation of the real temperature distribution in the sample, several temperature 
profiles were tested with additional sensors as shown in Figure 6. One sensor is positioned inside an LSR sample through a small hole to enable the temperature measurement inside the sample. Fiber optical sensors are used, which allows temperature measurements even during the polarization phase. For recording a fiber optical signal conditioner from FISO is used.

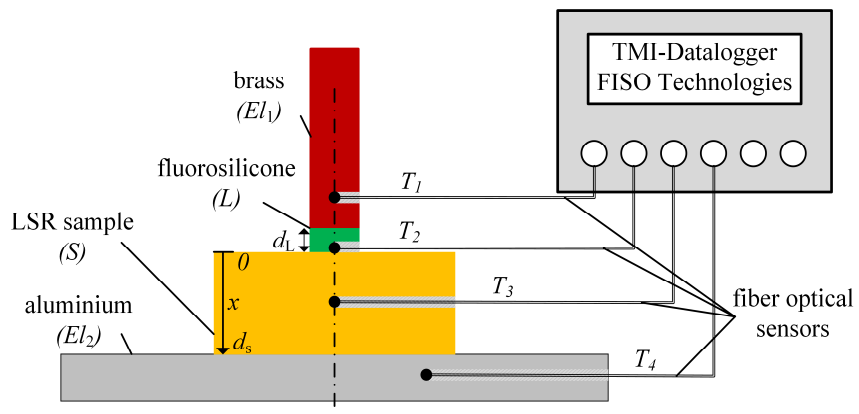

Figure 6: Setup for temperature measurement and control

In total three heating elements are evenly distributed at the top of electrode $E l_{2}$ (see Figure 1). The heating elements DN515-1228 are operated by a simple $12 \mathrm{~V} / 5 \mathrm{~A}$ DC power supply. A potentiometer is used to set the desired temperature of the heating elements. To achieve a stable temperature gradient the top electrode $E l_{1}$ is cooled or heated by transformer oil with the help of a bath thermostat from LAUDA. A high resistivity oil must be used to prevent a current flow through the bath thermostat. For this purpose the transformer oil Shell Diala S3$Z X 1$-dried is used. It must be mentioned that the temperature $T_{3}$ inside the electrode $E l_{2}$ is not measured exactly in the symmetry axis because the acoustic signal would be distorted by the required hole for the fiber optical sensor. Because of the high thermal conductivity of metals it is admissible to assume that the complete electrodes $E l_{1}$ and $E l_{2}$ are isothermal after reaching steady state conditions. For verification the electrode $E l_{1}$ was set to $20^{\circ} \mathrm{C}$ and $E l_{2}$ to $60{ }^{\circ} \mathrm{C}$. An LSR sample with a thickness $d_{\mathrm{s}}$ of $4 \mathrm{~mm}$ and a coupling layer with a thickness $d_{\mathrm{L}}$ of $1 \mathrm{~mm}$ were placed between $E l_{1}$ and $E l_{2}$ (see Figure 6). The measured temperature profiles are shown in Figure 7.

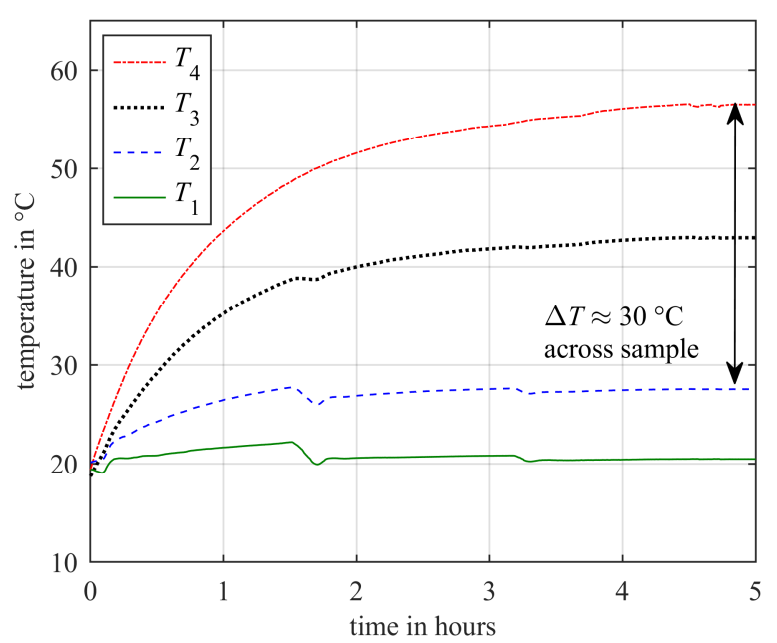

Figure 7: Measured temperature profiles
It can be seen that the final temperature of $E l_{2}$ is around $57^{\circ} \mathrm{C}$ and that of $E l_{1}$ around $21^{\circ} \mathrm{C}$. This outlines that a permanent temperature control of $E l_{1}$ and $E l_{2}$ is mandatory. The small deviation from the final temperatures in steady state condition originates from the heat capacitance of the whole setup. Furthermore, Figure 7 shows that the steady temperature distribution is achieved after 5 hours. The real temperature gradient across the sample is between $T_{2}$ and $T_{4}$ and is around $30{ }^{\circ} \mathrm{C}$ for this example. Due to the fact that the heat conductivity of the fluorosilicone layer and the investigated LSR samples are very similar, a linear temperature distribution across the sample is assumed. To verify the linear temperature distribution inside of the sample $T_{\mathrm{s}}$ can be described according to (4):

$$
T_{\mathrm{S}}(x)=\frac{T_{4}-T_{1}}{d_{\mathrm{s}}+d_{\mathrm{L}}} \cdot\left(x+d_{\mathrm{L}}\right)+T_{1}
$$

Taking the final stationary temperatures after 5 hours from Figure 7 and plotting them together with the profile according to (4) it can be seen that the linear temperature distribution is real. This relationship is indicated in Figure 8 where the blue line represents the temperature profile according to (4).

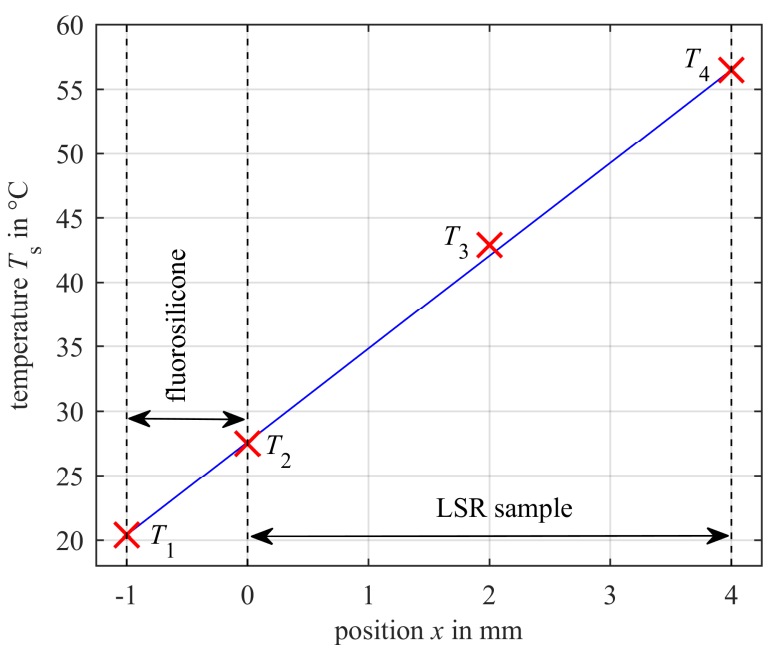

Figure 8: Verification of the linear temperature profile

Based on the verification a linear temperature profile according to (4) is assumed for all further investigations by using the measured temperatures $T_{1}$ and $T_{4}$ at the electrodes $E l_{1}$ and $E l_{2}$, respectively. It is just important to pay attention to the temperature drop across the fluorosilicone coupling layer $L$.

\section{Calibration process}

As it can be seen in Fig. 9 the main signal is distorted. The main influencing factors are the system response from the whole setup including the characteristics of the piezoelectric transducer, the amplifiers and the sample itself. The positive and negative overshoots are due to the mentioned system response from the whole PEA setup. To eliminate this effect the transfer function is determined in the frequency domain. To ensure that no space charge is responsible for the overshoots the sample must be discharged, and the signal $u_{\mathrm{m}}$ is recorded ten seconds after DC voltage application. When taking care of these points 
the transfer function can be determined. The detailed procedure is described in [4].

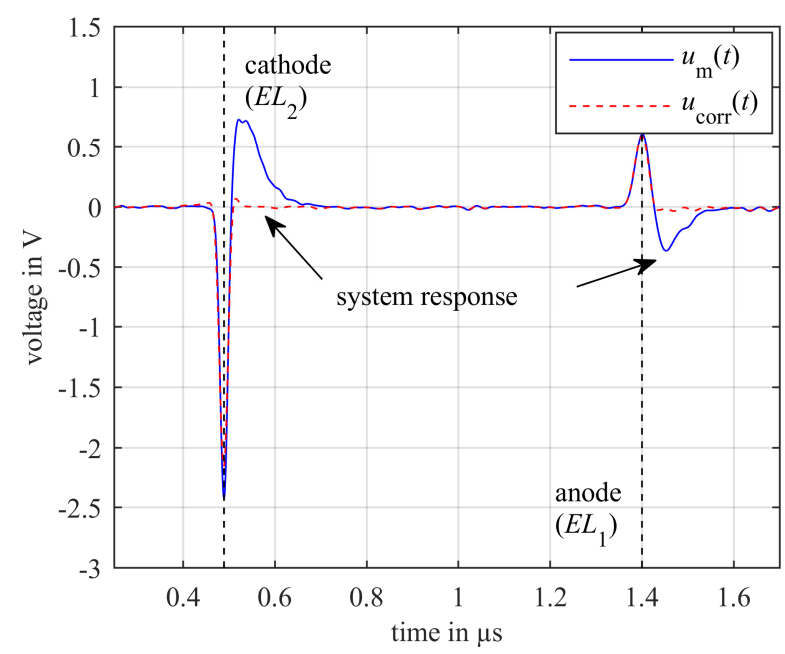

Figure 9: Elimination of the system response

The corrected signal $u_{\text {corr }}$ is also depicted in Fig. 9 but the second peak from the $\mathrm{HV}$ electrode $E l_{1}$ is still smaller and wider than the one from $E l_{2}$. This is due to the characteristics of the sample itself. Every material has a certain influence on the propagation of acoustic waves, which is described by the spectrum of the transmitted wave in the frequency domain according to $(5)[6,7]$.

$$
P(\omega, x)=P(\omega, 0) \cdot e^{-(\alpha(\omega)+\mathrm{i} \beta(\omega)) \cdot x}
$$

In (5) the attenuation factor $\alpha(\omega)$ describes the attenuation of the acoustic waves and the dispersion factor $\beta(\omega)$ describes the frequency dependence of the speed that causes the second peak to be wider. $P(\omega, x)$ is the spectrum of the wave at any position $x$ inside of the sample, and $P(\omega, 0)$ is the signal caused by induced charges at $E l_{2}$ where the wave has to travel just through $E l_{2}$ towards the transducer. As acoustic attenuation and dispersion of metals is negligible it can be assumed that the signal from $E l_{2}$ is identical to the original wave $P(\omega, 0)$. The transmitted wave $P(\omega, \mathrm{d})$ from $E l_{1}$ has to travel through the sample with a thickness of $d_{\mathrm{s}}$ and is distorted as shown in Fig. 9. Using the relation in (5) the factors $\alpha$ and $\beta$ can be calculated according to $(6 \& 7)$, assuming that $P(\omega, 0)$ is the spectrum of the original wave and $P(\omega, \mathrm{d})$ the one of the transmitted wave.

$$
\begin{gathered}
\alpha(\omega)=-\ln [|P(\omega, d)| /|P(\omega, 0)|] \cdot 1 / d_{\mathrm{s}} \\
\beta(\omega)=-[\varphi(\omega, d)-\varphi(\omega, 0)] \cdot 1 / d_{\mathrm{s}}
\end{gathered}
$$

In $(6 \& 7) \varphi$ is the phase and $|P|$ the amplitude of the signal spectrum. The attenuation and dispersion factors for LSR 1 and LSR 2 samples with a thickness of $1 \mathrm{~mm}$ are calculated for different temperature profiles. Two different isothermal conditions and one temperature gradient are analyzed, and the results are depicted in Figure 10. It is obvious that both the attenuation and the dispersion increase for higher frequencies. This can be interprated such that the higher frequency components of the acoustic waves are more attenuated and dispersed [8].
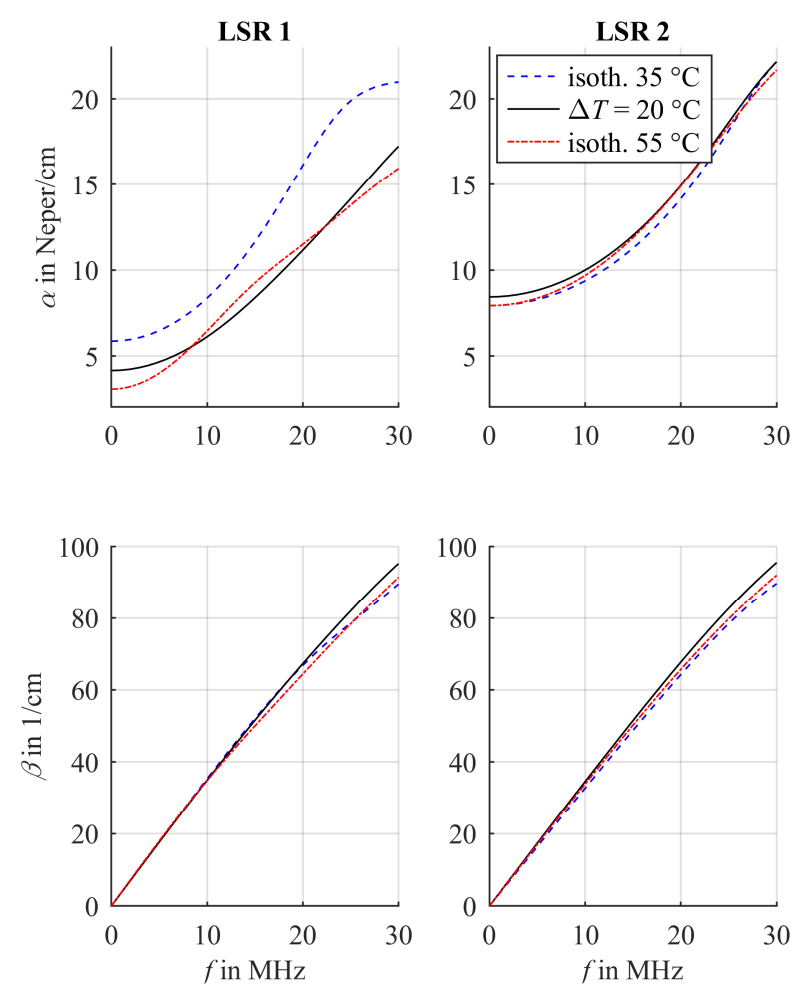
Figure 10: Attenuation and dispersion factors at different
temperatures for LSR 1 and LSR 2

As shown in Figure 10 the dispersion $\beta$ is nearly independent of the filler concentration and the temperature profile. The attenuation $\alpha$ is slightly increased in the case of LSR 2 samples indicating that the nanofillers are moderately hindering the acoustic waves. The reduced speed of LSR 2 is also shown in Table 1 verifying the observed behavior of $\alpha$. For LSR 1 it can be observed that both the attenuation and the speed decrease as the temperature increases. This effect is outlined more clearly in Figure 11 showing the measured voltage signals over time for different temperature profiles.
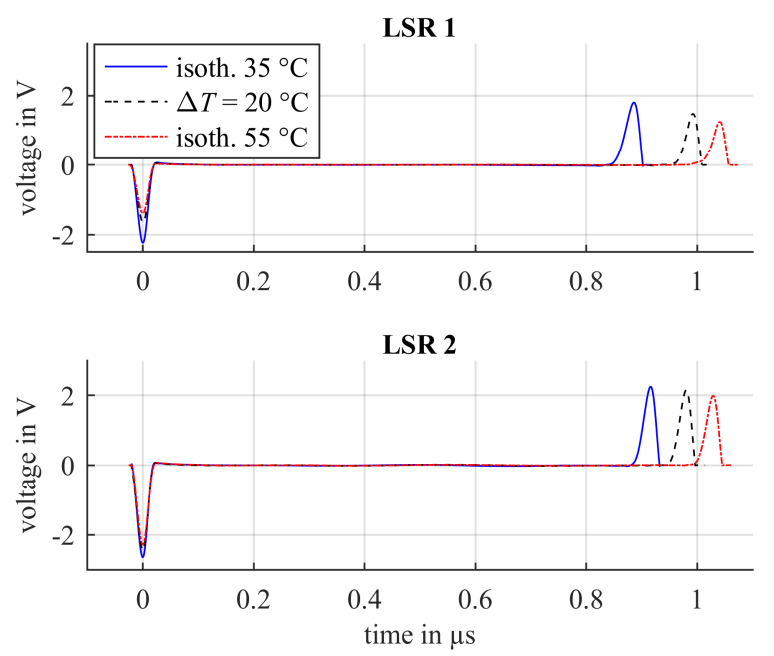

Figure 11: Influence of temperature on the speed of sound 
For both materials, LSR 1 and LSR 2, it is clearly visible that the second peak from the high voltage electrode $E l_{1}$ is delayed at higher temperatures. For all measurements the sample thickness was constant at $1 \mathrm{~mm}$. This assumption is permissible because the top electrode was fixed to the ground electrode $E l_{2}$ with screws to prevent any expansion in vertical direction of the LSR samples during heating. The reduced speed can be explained by the temperature influence of the shear modulus. The acoustic waves in elastic materials are transmitted by shear waves. According to $[8,9]$ the speed is proportional to the square root of the shear modulus and decreases for increasing temperatures. For more detailed explanations refer to $[8,9]$. Finally, the system response and the influence of attenuation and dispersion are eliminated as described and the final result is shown in Figure 12.

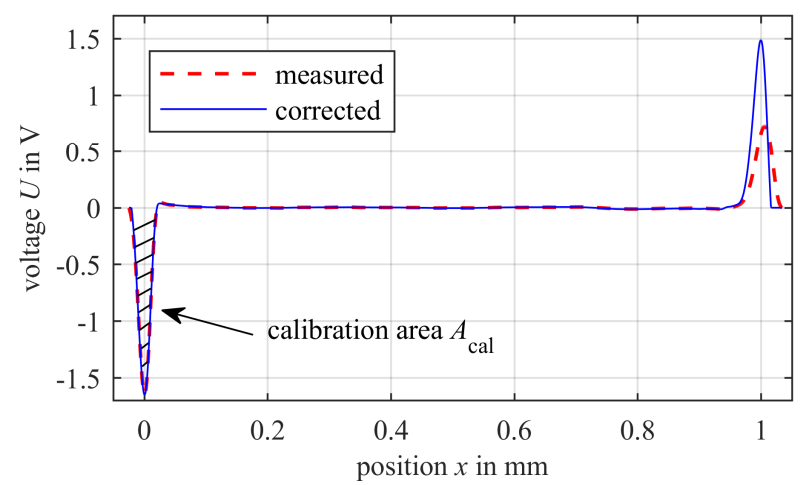

Figure 12: Elimination of attenuation and dispersion influence

To determine the charge density from the measured voltage signal the area $A_{\text {cal }}$ of the first peak representing the induced charge layer at $E l_{2}$ is taken to calculate the overall calibration factor $K_{\mathrm{cal}}$, by which the measured voltage signal $U_{\mathrm{m}}$ can finally be translated into the charge density $\rho$ according to $(8-10)$ $[3,10,11,12]$. The final result is shown in Figure 13, where in addition the profiles of the electric field and the electric potential distribution are calculated ten seconds after the application of a DC voltage of $10 \mathrm{kV}$ on a $1 \mathrm{~mm}$ LSR 2 sample.
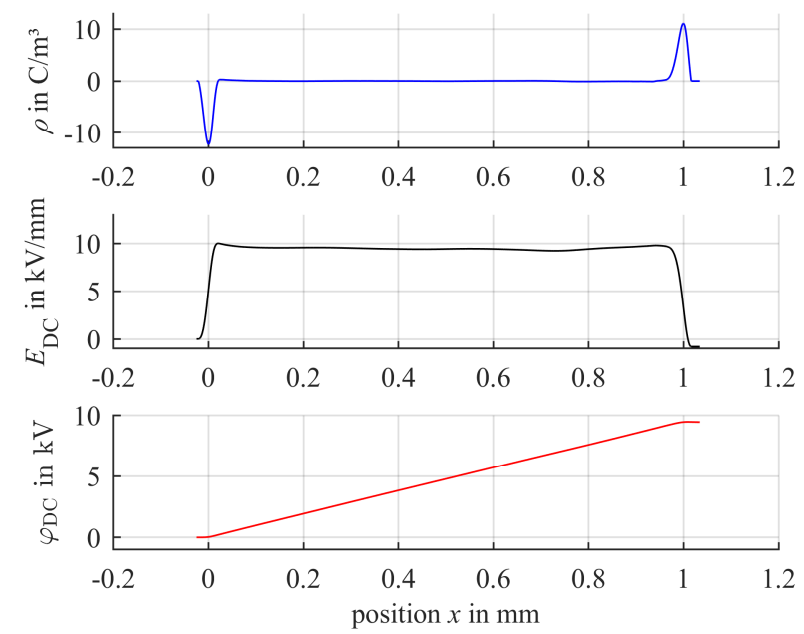

Figure 13: Charge density, field strength and potential distribution across LSR 2 at $10 \mathrm{kV}$ (after $10 \mathrm{~s}$ )

$$
\begin{gathered}
\rho=\frac{U_{\mathrm{m}}(x)}{K_{\mathrm{cal}}} \\
K_{\text {cal }}=\frac{d_{\mathrm{s}} \cdot A_{\mathrm{cal}}}{\varepsilon_{0} \varepsilon_{\mathrm{r}} \cdot U_{\mathrm{DC}}} \\
A_{\text {cal }}=\int U_{\text {cal }}(x) \mathrm{d} x
\end{gathered}
$$

\section{Conclusion}

In this work a PEA setup was developed and optimized to investigate LSR samples with Carbon Black nanofillers. The system includes temperature controlled electrodes to perform measurements closer to real operating conditions. It was found that for LSR both attenuation and dispersion must be considered. For this purpose both factors were analyzed at different temperature profiles and for different filler concentrations. The main result is that both factors should be determined for each material and temperature profile. The presented PEA system and the signal processing will be used to investigate the timedependent behavior of the space charge for different filler concentrations and temperatures in future work.

\section{References}

[1] G. Mazzanti, G. Marzinotto, Extruded Cables for High-Voltage DirectCurrent Transmission: Advances in Research and Development, IEEE Press, 2013.

[2] J. Xiang et al, "Space Charge Characteristics in XLPE/BN Nanocomposites at Different Temperatures", 12th Int. Conference on the Properties and Applications of Dielectric Materials, 952-955, 2018.

[3] A. Penttinen, "Design of Pulsed Electroacoustic Measurement System for Space Charge Characterisation", Thesis, Lappeenranta University of Technology, 2012.

[4] R Hussain, J. Moxter, V. Hinrichsen, "Development and optimization of a Pulsed Electroacoustic system suitable for silicone rubbers with carbon black nanofillers", Jicable - $10^{\text {th }}$ International conference on insulated power cables, in press, Paris, 2019.

[5] T. Takada et al, "Space charge measurement in dielectric and insulating materials - PEA, PWP and TSM methods", Cigré Task Force D1.12.01, February 2006.

[6] R. Bodega, "Space Charge Accumulation in Polymeric High Voltage DC Cable Systems", Thesis, Delft University Press, 2006.

[7] T. Wendel, J. Kindersberger, M. Hering, K. Juhre, "Space Charge measurement in epoxy according to the pulsed electro acoustic method under consideration of attenuation and dispersion", VDE Hochspannungstechnik Berlin, 451-456, 2018.

[8] D. W. Van Krevelen ${ }^{\dagger}$, K. Te Nijenhuis, Properties of Polymers - Their correlation with chemical structure; their numerical estimation and prediction from additive group contributions $4^{\text {th }}$ edition, Elsevier, 2009.

[9] P. H. Mott, C. M. Roland, R. D. Corsaro, "Acoustic and dynamic mechanical properties of a polyurethane rubber", Journal of Acoustical Society of America, DOI: 10.1121/1.1459465, April 2002.

[10]P. Morshuis, M. Jeroense, "Space Charge Measurements on Impregnated Paper: A Review of the PEA Method and a Discussion of Results", IEEE Electrical Insulation Magazine, vol. 13, 26-35, 1997.

[11]Y. Li, M. Yasuda, T. Takada, "Pulsed Electroacoustic Method for Measurement of Charge Accumulation in Solid Dielectrics", IEEE Transactions on Dielectrics and Electrical Insulation, vol. 1, 188-195, 1994.

[12] M. Jeroense, "Charges and Discharges in HVDC Cables: in particular in mass-impregnated HVDC cables”, Thesis, Delft University Press, 1997. 